\title{
A!
}

This is an electronic reprint of the original article.

This reprint may differ from the original in pagination and typographic detail.

Massel, Francesco; Leskinen, Mikko J.; Törmä, Päivi

\section{Hopping modulation in a one-dimensional Fermi-Hubbard Hamiltonian}

Published in:

Physical Review Letters

DOI:

10.1103/PhysRevLett.103.066404

Published: 07/08/2009

Document Version

Publisher's PDF, also known as Version of record

Please cite the original version:

Massel, F., Leskinen, M. J., \& Törmä, P. (2009). Hopping modulation in a one-dimensional Fermi-Hubbard

Hamiltonian. Physical Review Letters, 103(6), 1-4. [066404]. https://doi.org/10.1103/PhysRevLett.103.066404

This material is protected by copyright and other intellectual property rights, and duplication or sale of all or part of any of the repository collections is not permitted, except that material may be duplicated by you for your research use or educational purposes in electronic or print form. You must obtain permission for any other use. Electronic or print copies may not be offered, whether for sale or otherwise to anyone who is not an authorised user. 


\title{
Hopping Modulation in a One-Dimensional Fermi-Hubbard Hamiltonian
}

\author{
Francesco Massel, Mikko J. Leskinen, and Päivi Törmä* \\ Department of Applied Physics, P.O. Box 5100, 02015 Helsinki University of Technology, Finland
}

(Received 2 May 2009; published 7 August 2009)

\begin{abstract}
We consider a strongly repulsive two-component Fermi gas in a one-dimensional optical lattice described in terms of a Hubbard Hamiltonian. We analyze the response of the system to a periodic modulation of the hopping amplitude in the presence of a large two-body interaction. By (essentially) the exact simulations of the time evolution, we find a nontrivial double occupancy frequency dependence. We show how the dependence relates to the spectral features of the system given by the Bethe ansatz. The discrete nature of the spectrum is clearly reflected in the double occupancy after a long enough modulation time. We also discuss the implications of the 1D results to experiments in higher dimensional systems.
\end{abstract}

DOI: 10.1103/PhysRevLett.103.066404

Ultracold atomic gases systems couple weakly with the surrounding environment and are highly controllable [14]; therefore, they offer excellent possibilities to investigate the dynamics of strongly correlated quantum manybody systems. Much attention has been recently devoted to the study of the dynamical properties from both the experimental [5,6] and the theoretical [7-11] point of view. Especially, one-dimensional (1D) systems, accessible by experiments and theoretically exactly solvable in some cases, can be used to obtain a thorough understanding of the many-body ground state and the dynamics. In this Letter, we present an (essentially) exact time-evolving block decimation (TEBD) simulation of the dynamics of a repulsively interacting $1 \mathrm{D}$ system and reveal a nontrivial time dependence which we explain using the Bethe ansatz (BA). We extend the analysis also to the harmonically trapped case essential for ultracold gas experiments. In higher dimensions, the relevance of Mott and antiferromagnet (AFM) physics in connection to high- $T_{c}$ superconductivity (see, e.g., [12]) suggests that the investigation of the equivalent systems in the framework of ultracold gases, especially in two dimensions, may shed new light on high- $T_{c}$ superconductor physics. Our results are relevant for such experiments by showing - with analysis that does not assume mean-field approximation nor linear response-how the discrete nature of the spectrum is reflected in the dynamics of a lattice modulation experiment.

We examine the dynamical properties of a two-species ultracold atomic gas loaded in a 1D optical lattice both for open boundary conditions (OBC) and in the presence of parabolic confinement. In particular, we perform an (essentially) exact numerical simulation of this system when a periodic lattice modulation is applied. To this end, we consider the 1D Hubbard Hamiltonian, in the presence of an external parabolic confining potential

$$
H=H_{J}+H_{\mathrm{int}}+\sum_{i}^{L} V_{i}\left(n_{i \uparrow}+n_{i \downarrow}\right),
$$

where $H_{J}=-J \sum_{i, \sigma=\{\uparrow, \downarrow\}}^{L} c_{i \sigma}^{\dagger} c_{i+1 \sigma}+$ H.c., $H_{\text {int }}=U \sum_{i}^{L} n_{i \uparrow} n_{i \downarrow}$, $V_{i}=\Omega\left(i-i_{0}\right)^{2}, J$ is the hopping amplitude, and $U$ is the
PACS numbers: 71.10.Fd, 03.75.Ss, 78.90.+t

on-site interaction. In the limit $U / J \gg 1$, the only effect of the lattice modulation is a modulation of the hopping amplitude $J$, the effect on $U$ being negligible (see [11]).

We focus on the double occupancy (DO) expectation value $\left\langle n_{i \uparrow} n_{i \downarrow}\right\rangle$, when a (small) periodic modulation $\delta J \sin (\omega t)$ of the hopping amplitude $J$ is applied for a given time to the ground state of the Hamiltonian given in Eq. (1) for two different situations: $U / J=60, L=20$, and particle number $N_{p}=12$ and $U / J=20, L=40$, and $N_{p}=24$, respectively. Our simulation is performed with a TEBD algorithm [13,14], for both the ground-state calculation (imaginary-time evolution) and the real-time evolution. The numerical results show that the ground state is constituted by a central Mott region with one atom per site, surrounded by two small metallic (Luttinger liquid) regions where the filling is less than one. In order to avoid finite-size effects, we have considered a lattice size exceeding the actual extent of the atomic cloud by a few lattice sites. Heuristically, the Luttinger liquid phase corresponds to the regions where $\left\langle n_{i}^{2}\right\rangle-\left\langle n_{i}\right\rangle^{2} \neq$ const; see [15]. Moreover, the static structure factor $S(q)$ in the ground state of the finite systems here considered exhibits the same qualitative features of the 1D Heisenberg AFM chain (i.e., a slowly decaying peak centered around $q=\pi$ ), as expected in the $U \gg J$ limit of the Hubbard Hamiltonian [16].

For an infinite chain at half filling, if hopping and parabolic confinement are suppressed, the (highly degenerate) first excited state is represented by a site with an empty site and a doubly occupied one (henceforth particlehole excitation). The energy gap between this state and the ground state is equal to $U$. To investigate the spectral properties of the system when $U \gg J$, it seems then natural to choose $\omega \simeq U$ in $\delta J \sin (\omega t)$, with $\delta J / J=0.1$ throughout the Letter.

In Fig. 1 (inset), we show the DO as a function of frequency for short times, from which one notices that a broad peak appears as in correspondence of the value $\Omega=$ $U$, consistently with the value of the gap of the particlehole excitation. However, for larger times a richer structure appears (Fig. 1). From Fig. 2, it is clear how it is possible to 


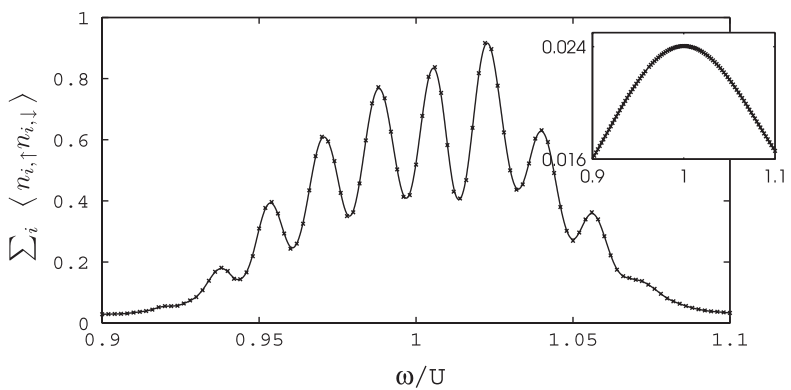

FIG. 1. Double occupancy $\sum_{i}\left\langle n_{i, \uparrow} n_{i, \downarrow}\right\rangle$ as a function of frequency for long ( $t=10$, main panel) and short ( $t=0.5$, inset) times. A single broad peak appears for $\omega / U \simeq 1(L=20$, $U / J=60, \Omega / J=0.1$.

distinguish between short- $\left(t<t^{*}\right)$ and long- $\left(t>t^{*}\right)$ time behavior; the explicit value of the threshold $t^{*}$ will be derived below.

The key idea in understanding the time dependence of $\left\langle n_{i \uparrow} n_{i \downarrow}\right\rangle$ is that the resolution of degeneracy depends on the modulation time scale. For $t<t^{*}$ it is possible to consider the particle-hole excited states as quasidegenerate; hence, the transition probability between the ground state and the quasicontinuum (centered around $U$ ) of excited states is given by $P(t) \propto \delta J^{2} \sin ^{2}[(U-\hbar \omega) t / 2 \hbar](U-\hbar \omega)^{2}$. The threshold time is then calculated as the time when the internal structure of the excited states band becomes visible, namely, $t^{*}=2 \pi / \Delta E$, where $\Delta E$ is the energy difference in the quasicontinuum of excited states which, in our case, will be determined by the BA. For $t>t^{*}$, the transition probability is given by

$$
\sum_{n, E_{n} \simeq E_{\text {g.s. }} \pm \omega} P_{n}(t)=\left.\left(\frac{2 \pi}{\hbar}\right)\left|V_{n, \text { g.s. }}\right|^{2} \rho\left(E_{n}\right) t\right|_{E_{n} \simeq E_{\text {g.s. }} \pm \hbar \omega},
$$

where $E_{n}$ represents the energy of one of the quasidegenerate excited states. The numerical results plotted in Fig. 2 show how the transition between these two regimes is quite abrupt, allowing an easy comparison with the theoretical

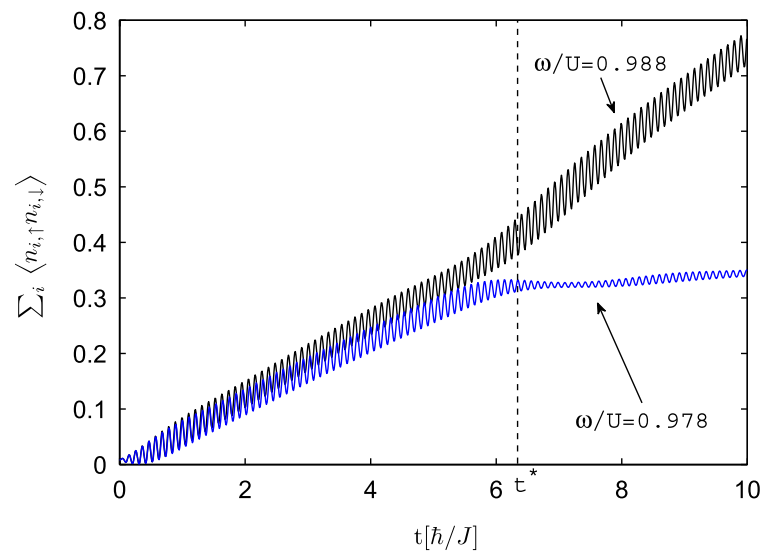

FIG. 2 (color online). Double occupancy $\sum_{i}\left\langle n_{i, \uparrow} n_{i, \downarrow}\right\rangle$ as a function of time, for two different frequency values corresponding to a minimum $(\omega / U=0.978)$ and a maximum $(\omega / U=0.988)$ of the plot in Fig. 1 (same parameters as Fig. 1). value of $t^{*}$. Equation (2) explains the piecewise linear time dependence of DO, which is induced by the different effective density of states $\rho\left(E_{n}\right)$ and perturbation matrix elements $\left|V_{n, \text { g.s. }}(\delta J)\right|^{2}$ before and after $t^{*}$. In particular, in order to have a peak in the DO spectrum, both the perturbation matrix element and the density of states at the appropriate energy must be different from zero, suggesting that the DO spectrum exhibits selection rules in its longtime peak distribution. In the two-site problem, it is possible to see explicitly how the ground state with two particles, represented by singlet state $|S\rangle=(1 / \sqrt{2})(|\uparrow, \downarrow\rangle-$ $|\downarrow, \uparrow\rangle)$, is coupled by the hopping modulation to the state $\left|D_{+}\right\rangle=(1 / \sqrt{2})(|\uparrow \downarrow, 0\rangle+|0, \uparrow \downarrow\rangle)$ only and not to the state $\left|D_{-}\right\rangle=(1 / \sqrt{2})(|\uparrow \downarrow, 0\rangle-|0, \uparrow \downarrow\rangle)$.

The 1D nature of the problem allows some insight on the peak position deriving from the exact (BA) solution of the 1D Hubbard Hamiltonian. As a first step, we make contact between the BA and the numerical solution of a small linear chain with open boundary conditions $(\Omega=0)$ and $U / J \rightarrow \infty(U / J=500)$. To this end, we consider the BA equations for an open Hubbard chain (see [17] and supplementary material [18]). The solution of BA equations with respect to the charge momenta $k_{j}$ and spin rapidities $\lambda_{\alpha}$ allows one to determine energy and momentum eigenstates whose values can be expressed in terms of $k_{j}$ as

$$
E=-2 J \sum_{j=1}^{N} \cos \left(k_{j}\right), \quad P=\left[\sum_{j=1}^{N} k_{j}\right] \bmod 2 \pi .
$$

Along the lines of the derivation by Ogata and Shiba [19] applied to the case of $\mathrm{OBC}$, Bethe-ansatz equations lead to the simple relation $k_{j}=\pi I_{j} /(L+1)$, in the limit $U / J \rightarrow \infty$. The latter expression is particularly relevant since it describes the spectrum of the considered Hamiltonian in terms of spinless fermionic particles. This result can be interpreted within the general framework of spin-charge separation of excitations in 1D systems (see, e.g., [20]). In particular, the ground state for $N=L$ is obtained when $I_{j}=1, \ldots, N$. Its energy is given by $E=$ $-2 J \sum_{j=1}^{L} \cos \left(k_{j}\right)$, and the Fermi quasimomentum is given by $k_{F}=\pi L /(L+1)$. The excitations of the system can be described in terms of particle-hole excitations of this system. Hence, since the first Hubbard band is full,

$$
\tilde{\Delta} E=\Delta E+U=-2 J\left[\cos \left(k_{p}\right)-\cos \left(k_{h}\right)\right]+U,
$$

where $E_{p}=-2 J \cos k_{p}+U$ corresponds to the energy of an extra particle added in the second Hubbard band and $2 J \cos \left(k_{h}\right)$ the energy of the hole in the first Hubbard band. Since having a particle and a hole in the same momentum state would not contribute to the increase of the interaction energy by $U$, these states must not be considered in the calculation of the energy-level structure around $U$. We also note that, in the thermodynamic limit $L \rightarrow \infty$, the discrete energy-level structure becomes a continuous band of width $8 \mathrm{~J} / U$, as it is possible to deduce from Eq. (4). Our simulations are at $T=0$, but the features in the spectra are 

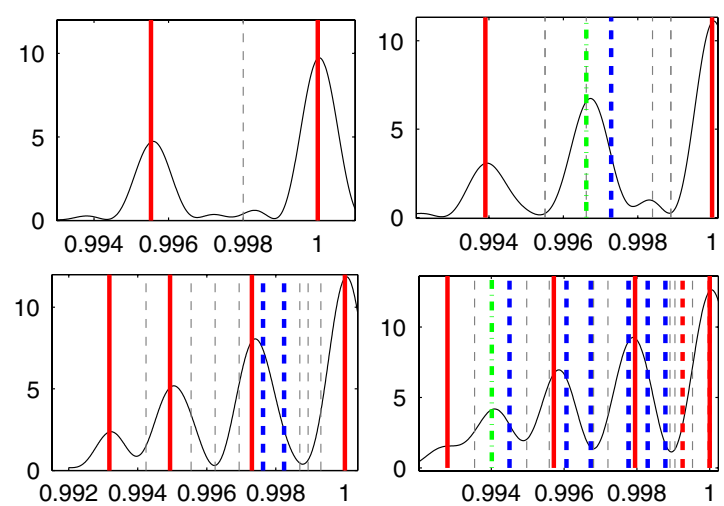

FIG. 3 (color online). DO spectrum for $U / J=500$ and $L=$ $4,6,8,10$ (top left to bottom right). Vertical lines represent the value $\tilde{\Delta} E$ for various $k_{p}$ and $k_{h}$. Red continuous lines represent the values listed in Table I. Blue dashed lines represent transitions between states of even parity (i.e., $i_{p}$ and $i_{h}$ even), which do not seem to correspond to a peak in the DO spectrum. Gray dotted lines represent states with $i_{p}=2 n+1$ or $i_{h}=2 n+1$, with $n \in \mathbb{Z}$. Green dashed-dotted lines represent a particular condition of coincidence of the frequency between a "red line" transition and a "gray line" transition with $i_{p}=2 n+1, i_{h}=$ $2 n^{\prime}+1$, and $i_{p}-i_{h}=2 n^{\prime \prime}$ (see Table I). For ease of reading, the right part of the spectrum has been omitted due to its symmetry around $\omega=1$ (cf. the slight asymmetry in the trapped case, Fig. 1). $x$ axis: $\omega / U ; y$ axis: $\left\langle n_{i \uparrow} n_{i \downarrow}\right\rangle$.

expected to be visible if the system is in the Mott state and $T<\tilde{\Delta} E$.

We have made a connection to the particle-hole excitation spectrum by performing hopping modulation on systems with open boundary conditions (i.e., no parabolic confinement), with $U / J=500$ and $L=4,6,8,10$. As seen in Fig. 3, the DO peaks correspond to specific particle-hole excitations, supporting the explanation of the DO spectrum in terms of selection rules (see Table I). Even if the simulations in the presence of a parabolic confining potential with $U / J=20,60$ cannot be described exactly within the above formalism, we suggest that it is possible to make contact between a description in terms of spinless fermions and the numerical results, the only effect of parabolic confinement being the different spectrum in the Mott phase. This hypothesis is justified by the nature of the metallic phase in the limit $U / J \gg 1$ (gapless spectrum, small spatial extent) and confirmed by numerical evidence. As in the case where the parabolic confining potential is absent, we assume that the spectrum of the system can be described in terms of particle-hole excitations of spinless fermions moving in a lattice, in the presence of a global parabolic confinement.

In Ref. [21], it has been shown that, for $4 J \geqslant \Omega$, the approximate description of the single-particle spectrum can be carried out in terms of low-energy excitations with quantum number $n<n_{c}, n_{c}=2\|\sqrt{2 J \Omega}\|-1$, where $\|x\|$ is the integer closest to $\|x\|$, and high-energy excitations with $n>n_{c}$. If $n<n_{c}$, the single-particle energy is
TABLE I. Comparison of theory and numerics.

\begin{tabular}{lrrcc}
\hline \hline & $i_{p}$ & $i_{h}$ & $\tilde{\Delta} E / U$ & $\left(\tilde{\Delta} E-\omega_{p k}\right) / U$ \\
\hline$L=4$ & 4 & 4 & 1.0000 & $<10^{-4}$ \\
& 2 & 4 & 0.9955 & $<10^{-4}$ \\
& $(1$ & 3 & 0.9955 & $\left.<10^{-4}\right)$ \\
$L=6$ & 6 & 6 & 1.0000 & $<10^{-4}$ \\
& 2 & 4 & 0.9966 & $\simeq 10^{-4}$ \\
& $(3$ & 5 & 0.9966 & $\left.\simeq 10^{-4}\right)$ \\
& 2 & 6 & 0.9939 & $<10^{-4}$ \\
& 8 & 8 & 1.0000 & $<10^{-4}$ \\
& 4 & 6 & 0.9973 & $\simeq 10^{-4}$ \\
& 2 & 6 & 0.9949 & $<10^{-4}$ \\
& 3 & 7 & 0.9949 & $\left.<10^{-4}\right)$ \\
& 2 & 8 & 0.9931 & $<10^{-4}$ \\
$L=10$ & 10 & 10 & 1.0000 & $<10^{-4}$ \\
& 6 & 8 & 0.9979 & $<10^{-4}$ \\
& 4 & 8 & 0.9957 & $\simeq 2 \times 10^{-4}$ \\
& 2 & 8 & 0.9940 & $<10^{-4}$ \\
& 3 & 9 & 0.9940 & $\left.<10^{-4}\right)$ \\
& 2 & 10 & 0.9928 & $<10^{-4}$ \\
\hline \hline
\end{tabular}

given by

$$
\begin{aligned}
E_{n}-E_{0}= & 2 \sqrt{J \Omega}(n+1 / 2)-\frac{\Omega}{32}\left[(2 n+1)^{2}+1\right. \\
& \left.-\frac{(2 n+1)^{3}+3(2 n+1)}{32 \sqrt{J / \Omega}}\right],
\end{aligned}
$$

where $E_{0}$ is a constant energy term. If, on the other hand, $n \geq n_{c}$, for the high-energy modes the energy eigenvalues are given by

$$
E_{n=2 i} \simeq E_{n=2 i-1} \simeq \Omega i^{2}+\frac{2 J}{(2 i)^{2}-1},
$$

representing states close to position eigenstates.

In the trapped system, performing a calculation analogous to the case with open boundary conditions, it is now

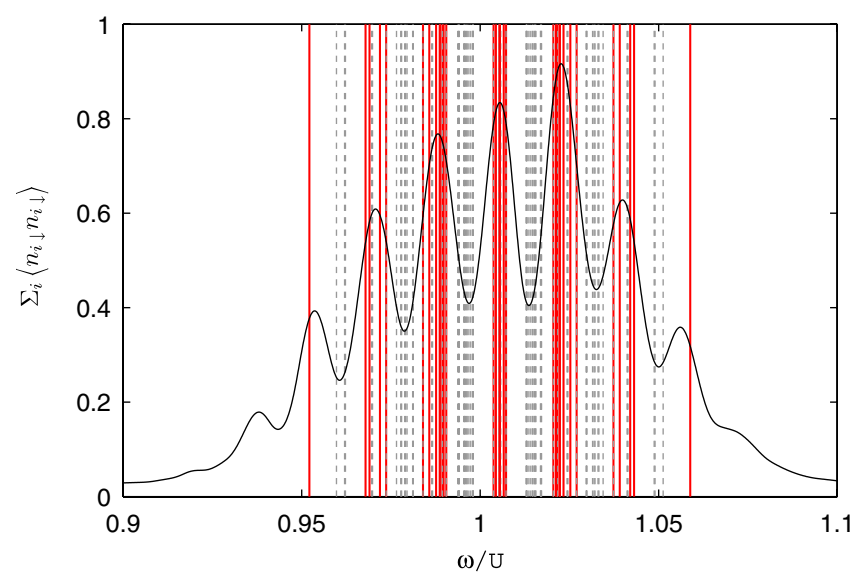

FIG. 4 (color online). Trapped system: energy levels (continuous red lines correspond to even values of $i_{p}$ and $i_{h}$ ) for $L=20$, $N_{\text {tot }}=12, U / J=60$, and $\Omega / J=0.1$. The peaks at the edge of the diagram do not correspond to any peak due to the approximate description of the energy levels. 


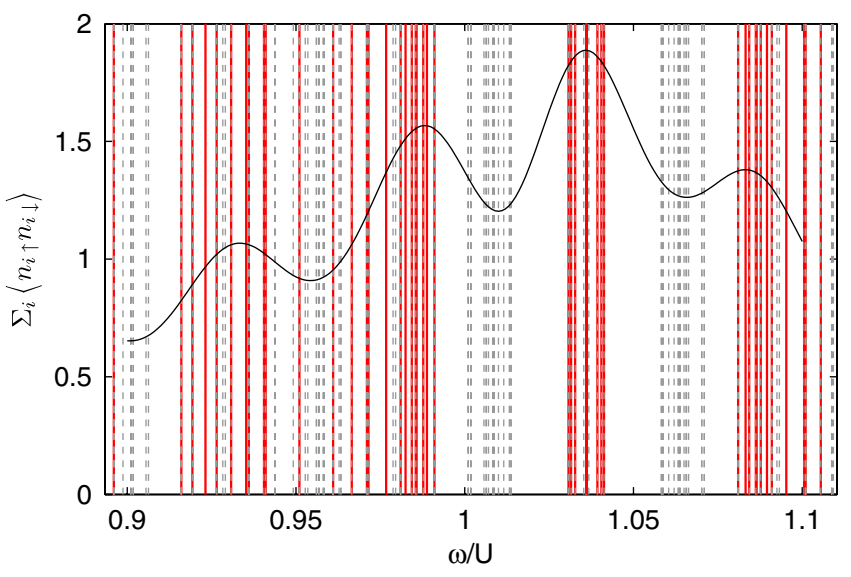

FIG. 5 (color online). Trapped system: energy levels (same definitions as in Fig. 4) $L=40, N=24, U=20$, and $\Omega / J=$ 0.1 (right), when values of $i_{h}$ and $i_{p}$ up to 20 are considered.

possible to compare the particle-hole excitation spectrum to the DO spectrum obtained numerically (see Figs. 4 and 5). It must be noted that, in Fig. 4, we have shifted $(\Delta \omega / U \simeq 0.667)$ the energy spectrum calculated in Eqs. (5) and (6), in order to obtain the superposition with the DO spectrum. The shift is due to a finite value of $U$. This effect can be explicitly calculated in the two-site case, where the energy-level splitting can be obtained analytically. In the case $U / J=60$, the DO spectrum shows good agreement with the spectrum from Eqs. (5) and (6), when we enforce the condition $n_{p}=$ even, $n_{h}=$ even.

Our analysis of the dynamical properties of a 1D Hubbard chain is in close relation to the experimental [5] and theoretical [11] investigations of Mott and AFM phases in optical lattices. Even if in our simulations there is no proper AFM phase, due to the dimensionality of the system, it is possible to relate the results obtained here concerning the peaks in the DO spectrum to those appearing in Ref. [11]. In the presence of AFM ordering, an energy gap $\propto J^{2} / U$ develops. In our case, the separation between the different energy levels, which mimics the effects of an energy gap, is induced either by the parabolic confinement or by finite-size effects. The qualitative similarity between the spectrum obtained here and in Ref. [11] confirms the relationship between the presence of finite energy gaps and the peaks in the DO spectrum. In our case, especially for the $\mathrm{OBC}$ case and for $U=60$, we are able to suggest how the excitation spectrum together with the nature of the lattice modulation determines the structure of the peaks. While Ref. [11] considers a higher dimensional system thus closer to the present experiments, our study is complementary in the sense that it does not assume mean-field approximation or linear response.

In addition, when performing a DO modulation experiment aiming at the observation of the spectrum depicted in Fig. 1, the effect of a finite modulation time must be taken into account. If we consider experimentally relevant values (see [5]) of $U, J$, and the modulation time $t_{\text {mod }}(U \simeq 5 \mathrm{kHz}$,
$\left.J \simeq 0.1 \mathrm{kHz}, \quad t_{\text {mod }}=50 / U=0.01 \mathrm{~s}\right)$, we obtain $t^{*} \simeq$ $2 \pi U / J^{2} \simeq 3.14 \mathrm{~s}$. Since this is an experimentally rather long time, the estimate suggests that smaller values of $U / J^{2}$ might be necessary to observe the AFM gap. In this calculation of $t^{*}$ we have considered $\Delta_{\mathrm{AFM}} \simeq J^{2} / U$.

In summary, with the aid of a TEBD numerical simulation, we have analyzed the properties of a fermionic gas in a 1D optical lattice, in the presence of parabolic confinement. The numerical results show that, for a sufficiently long time, a nontrivial peak structure appears in the DO spectrum. The peak structure has been qualitatively justified in terms of the BA solution of a chain with open boundary conditions, in the limit of $U / J \rightarrow \infty$. The results we have obtained, while not being a direct evidence of what should be observed in a modulation experiment in 3D [5] when $T<T_{\text {Néel }}$ [22], due to the different nature of the gaps in 3D and our 1D systems, explain in detail how the discrete nature of the spectrum is reflected in the lattice modulation experiment. The results thus suggest that the gap in the AFM phase can be observed via a nontrivial peak structure.

This work was supported by Academy of Finland and EuroQUAM/FerMix (Projects No. 213362, No. 217041, No. 217043, and No. 210953) and conducted as part of a EURYI scheme grant [23].

*paivi.torma@hut.fi

[1] D. Jaksch and P. Zoller, Ann. Phys. (N.Y.) 315, 52 (2005).

[2] C. Chin et al., arXiv:0812.1496.

[3] D. Jaksch et al., Phys. Rev. Lett. 81, 3108 (1998).

[4] S. Peil et al., Phys. Rev. A 67, 051603(R) (2003).

[5] R. Jordens et al., Nature (London) 455, 204 (2008).

[6] U. Schneider et al., Science 322, 1520 (2008).

[7] C. Kollath et al., Phys. Rev. A 74, 041604(R) (2006).

[8] K. Winkler et al., Nature (London) 441, 853 (2006).

[9] M. Cramer et al., Phys. Rev. Lett. 100, 030602 (2008).

[10] A. Flesch et al., Phys. Rev. A 78, 033608 (2008).

[11] R. Sensarma et al., arXiv:0902.2586.

[12] K. L. Hur and T. M. Rice, Ann. Phys. (N.Y.) 324, 1452 (2009).

[13] G. Vidal, Phys. Rev. Lett. 91, 147902 (2003).

[14] G. Vidal, Phys. Rev. Lett. 93, 040502 (2004).

[15] M. Rigol et al., Phys. Rev. Lett. 91, 130403 (2003).

[16] F.H.L. Essler et al., The One-Dimensional Hubbard Model (Cambridge University Press, Cambridge, England, 2005).

[17] H. Asakawa and M. Suzuki, J. Phys. A 29, 225 (1996).

[18] See EPAPS Document No. E-PRLTAO-103-077934 for supplementary material on Bethe-ansatz equations for open-boundary conditions. For more information on EPAPS, see http://www.aip.org/pubservs/epaps.html.

[19] M. Ogata and H. Shiba, Phys. Rev. B 41, 2326 (1990).

[20] T. Giamarchi, Quantum Physics in One Dimension (Oxford University, New York, 2003).

[21] A. M. Rey et al., Phys. Rev. A 72, 033616 (2005).

[22] J. Néel, Ann. Phys. (Paris) 5, 232 (1936).

[23] http://www.esf.org/euryi. 